\title{
Influencia del liderazgo auténtico en el estado emocional de militares desplegados en operaciones en el exterior: Estudio de la moral como variable mediadora
}

\author{
Pastor-Álvarez A. ${ }^{1}$, Molero-Alonso F. ${ }^{2}$, Moriano-León J.A. ${ }^{3}$
}

Sanid. mil. 2021; 77 (2): 73-80, ISSN: 1887-8571

\begin{abstract}
RESUMEN
Antecedentes: La influencia que los despliegues en operaciones militares tienen sobre la salud mental y el bienestar de los militares desplegados, ha sido siempre un tema de interés, que goza de bastante evidencia empírica. Algunas de las variables abordadas en el estudio de dicha influencia, han sido: el liderazgo y la moral. Objetivos: el presente estudio tendrá un triple objetivo. Por un lado, se analizará el efecto del Liderazgo Auténtico en el Estado Emocional de los soldados, durante un despliegue militar en misión de paz. Por otro, se observa si existe algún tipo de mediación del nivel de Moral, en la relación del liderazgo con el tipo de emocionalidad. Además, se observará si la acumulación de estrés durante el desarrollo de la misión afecta de algún modo a las relaciones planteadas anteriormente. Método: se recopilaron datos de 150 militares en el primer y quinto mes de misión, utilizando los cuestionarios ALQ, MORAL y PANAS; se utilizaron análisis de regresión, mediación y t-student para la comprobación de las hipótesis. Resultados: se ha confirmado la relación entre el Liderazgo Auténtico con el Estado Emocional, así como el efecto mediador de la Moral en las anteriores relaciones cuando las emociones son positivas. Se descartó en este estudio que hubiera diferencias significativas entre los datos del inicio y final de la misión. Conclusiones: el Liderazgo Auténtico se consolida como un modelo de gran relevancia en entornos militares, que va de la mano de la Moral en el beneficio del bienestar emocional de los militares desplegados.
\end{abstract}

PALABRAS CLAVE: Liderazgo auténtico, moral, estado emocional, despliegues militares.

\begin{abstract}
Influence of authentic leadership on the emotional state of deployed military personnel: A study of morale as a mediator variable. SUMMARY

Background: The influence of military operations on the mental health and well-being of the personnel deployed, has always been a topic of interest, supported by considerable empirical evidence. Some of the variables related with such influence have been leadership and morale. Objectives: this study will have a triple objective. On the one hand, the effect of Authentic Leadership on the Emotional State of soldiers will be analyzed during a peacekeeping mission. On the other hand, any type of mediation from the Moral level in the relationship of leadership with the type of emotionality will be observed. In addition, this study will check the accumulation of stress during the development of the mission affects in any way the previous mentioned relationships. Method: data were collected from 150 military ones, in the first and fifth months of the mission, using the ALQ, MORAL and PANAS questionnaires; Regression, mediation and t-student analysis were used to test the hypotheses. Results: the relationship between Authentic Leadership and the Emotional State was confirmed, as well as the mediating effect of Moral on previous variables when emotions were positive. No significant differences between the data at the beginning and end of the mission were found. Conclusions: Authentic Leadership is consolidated as a model of great relevance in military environments, which goes hand in hand with Moral, in benefit of the emotional well-being of military deployed personnel.
\end{abstract}

KEY WORDS: Authentic leadership, moral, emotional state, military deployments.

\section{INTRODUCCIÓN}

La influencia que los despliegues en operaciones militares tienen sobre la salud mental y el bienestar de los militares

1. Capitán Psicólogo destinado en la Sección de Psicología del CGUME

2. Catedrático del Departamento de Psicología Social y de las Organizaciones, y Vicedecano de Investigación y Transferencia de la Facultad de Psicología de la UNED.

3. Profesor asociado del Departamento de Psicología Social y de las Organizaciones de la Facultad de Psicología de la UNED.

Dirección para correspondencia: Cap. Psicólogo Alberto Pastor Álvarez. Sección de Psicología del CGUME. Base Aérea de Torrejón, A2 km 22. CP. 28850 Torrejón de Ardoz (MADRID)

Recibido: 22 de octubre de 2020

Aceptado: 21 de enero de 2021

doi: $10.4321 /$ S1887-85712021000200002 desplegados, ha sido siempre un tema de interés, sobre todo en aquellos escenarios de mayor intensidad física o emocional. $\mathrm{Si}$ bien el tema adquirió mayor relevancia a raíz de la inclusión en 1980, por parte de la Asociación Americana de Psiquiatría, del trastorno por estrés postraumático (TEPT) en su manual diagnóstico DSM-III, influenciados por las investigaciones con veteranos de la Guerra del Vietnam ${ }^{1}$. Tras los despliegues en conflictos más recientes, a partir principalmente de la Guerra del Golfo y las intervenciones militares en Irak y Afganistán; el interés de los investigadores por analizar las consecuencias en la salud mental del personal militar combatiente o desplegado se ha visto incrementado exponencialmente ${ }^{2,3}$.

Existe evidencia sobre las causas que originan el desgaste psicológico de los soldados desplegados, que en el desempeño de sus misiones se pueden encontrar con eventos potencialmente trau- 


\section{Pastor-Álvarez A., Molero-Alonso F., Moriano-León J.A.}

máticos como sufrir ataques, ver a compañeros heridos o muertos, o llegar a tener que manejar cadáveres o cuerpos mutilados ${ }^{4}$ La exposición a este tipo de experiencias de combate se ha relacionado con problemas de salud mental y desafíos relacionados tras los despliegues $^{5}$, manteniendo una relación estrecha con los síntomas del TEPT y la depresión ${ }^{6,7}$, así como con comportamientos de riesgo ${ }^{8}$ como el abuso de alcohol ${ }^{9}$. Además, la exposición al combate se asocia con un aumento de los síntomas somáticos, que pueden pasar más desapercibidos por parte de los servicios de salud mental, como pueden ser el dolor de estómago, dolor de cabeza, problemas de espalda o dolores musculoesqueléticos o náuseas ${ }^{10}$.

Pero más allá de las patologías clínicas específicas del ámbito de la salud, los despliegues militares afectan al estado emocional y bienestar de los soldados en relación con su entorno familiar y/o social. Las misiones internacionales provocan una tensión considerable en las familias militares. El aumento de las ausencias y la repetición de despliegues en determinados periodos de tiempo pueden aumentar la probabilidad de que aparezcan eventos familiares estresantes ${ }^{11}$, lo que puede provocar una vulnerabilidad en la estabilidad emocional del personal desplegado, y lo que es peor, dar lugar a situaciones de ruptura y conflicto, como el divorcio, durante el desarrollo o finalización de la misión. En el ámbito puramente laboral también encontramos estresores que pueden incidir en el agravamiento o mitigación de la situación de bienestar del personal. El riesgo de no estar en condiciones de cumplir con las misiones establecidas, o que los problemas de adaptación puedan conllevar consecuencias laborales, suponen una de las principales preocupaciones que en el medio o largo plazo generan una angustia añadida en el personal que comienza a padecer problemas de tipo psicológico. Como ejemplo encontramos el caso de las Fuerzas Armadas Españolas en Herat (Afganistán) ${ }^{12}$, donde un $19,08 \%$ del personal al que se le prestó atención en el Gabinete de Psicología del ROLE-2, requirió su repatriación o adelanto de su regreso a territorio nacional, habiendo sido los trastornos de ansiedad y adaptativos los diagnosticados con más frecuencia.

Por lo tanto, debemos tener presente que la preservación de la salud mental y del estado emocional del personal desplegado debe de tener un papel protagonista en todas las fases del planeamiento y ejecución de las operaciones militares. La cantidad y variabilidad de vicisitudes psicológicas y sociales que rodean a este personal, tanto en el transcurso de las misiones, como en la readaptación a Territorio Nacional (TN) producen una serie efectos psicológicos que pueden persistir durante un prolongado periodo de tiempo tras el regreso a casa ${ }^{13,14}$. Además, se debe tener en cuenta que la sintomatología puede ser demorada y tardar en aparecer tiempo después de haber finalizado la operación en el exterior ${ }^{15,16,17}$.

Existe un cierto acuerdo entre los expertos sobre que los despliegues militares per se pueden afectar a la salud y al estado emocional de los soldados ${ }^{18,19}$, incluso en aquellas operaciones donde el nivel de intensidad y estrés, así como el posible impacto psicológico, sea leve o moderado ${ }^{20}$. Por lo tanto, es importante desarrollar investigaciones que ayuden a determinar qué variables son inherentes al desarrollo de desajustes psicopatológicos en ambientes militares operativos, así como qué factores de protección pueden amortiguar o mitigar los efectos de una exposición prolongada al estrés operacional propio de las misiones internacionales. En los últimos años, sobre todo a raíz de la aparición del programa Comprehensive Soldier and Family Fitness (CSF2) ${ }^{21}$ del Ejército Estadounidense, se ha comenzado a estudiar y a promover el papel de la resiliencia como factor clave en la preservación y ajuste psicológico del personal. Dicho programa plantea un enfoque proactivo de prevención primaria en cuanto al estado emocional de los soldados; así como una orientación más reactiva, basada más en modelos de prevención secundaria y/o terciaria, hacia los problemas aparecidos como consecuencia de los despliegues en el exterior. La resiliencia es considerada una característica individual y un proceso $^{22}$, donde la historia personal, las estrategias de evaluación y afrontamiento, el apoyo social, la cohesión grupal y las cualidades del líder son aspectos importantes ${ }^{23,24}$. Es en este último aspecto, el liderazgo, en el que nos queremos centrar en nuestro estudio, ya que las conductas de los líderes juegan un importante papel, ya sea amortiguando o potenciando los efectos de los diferentes estresores sobre el bienestar de los empleados ${ }^{25}$.

Los jefes, cuando ejercen como figuras de liderazgo, juegan un rol fundamental pudiendo influir tanto en las conductas de salud de las organizaciones, como en las de los subordinados ${ }^{26}$. Las acciones positivas de los líderes pueden mejorar el ambiente laboral, y tener una influencia directa y positiva tanto en la satisfacción como en la implicación de los trabajadores, afectando directamente a su rendimiento y compromiso con la organización ${ }^{27}$. Por el contrario, los líderes negativos o tóxicos suelen dar mal ejemplo, creando un contexto en el que los miembros del grupo son menos compañeros entre sí. Como resultado de la disminución de la cohesión de la unidad, los individuos ven deterioradas sus actitudes hacia el servicio, lo que redunda en una menor satisfacción y compromiso ${ }^{28}$. Las consecuencias negativas a nivel psicológico, para aquellos que sufren en su día a día un tipo de liderazgo destructivo (por ejemplo, supervisión tóxica, abusiva, pequeña tiranía) son de gran alcance, significándose en un mayor nivel de estrés, posible abuso de alcohol y baja autoestima, en general2 ${ }^{29,30}$. Desafortunadamente, el impacto de los líderes tóxicos no se detiene en el individuo ${ }^{28}$, ya que también puede afectar de manera significativa a las relaciones personales del personal que lo sufre, incidiendo negativamente en las relaciones de pareja ${ }^{31}$, lo que genera un incremento de conflicto en el eje familia-trabajo ${ }^{32}$, que en el medio-largo plazo generará una carga de estrés que irá en detrimento de los recursos del personal, más aún cuando se encuentren desplegados en misiones de paz o de combate.

Podemos considerar entonces al liderazgo como una variable fundamental en la gestión del estrés del personal desplegado, ya sea como factor de protección, o como causa principal de éste ${ }^{27}$. La potenciación de los factores de liderazgo positivos tendrá un efecto beneficioso sobre el rendimiento, la eficacia, el bienestar, la satisfacción, la salud o la moral de los soldados. Los líderes están en una posición única para dar forma a las experiencias estresantes que los miembros del grupo entienden. El líder que, a través del ejemplo y la discusión, realiza una reconstrucción positiva de las experiencias estresantes compartidas, puede ejercer una influencia en todo el grupo en la dirección de su interpretación de la experiencia $^{33}$. Se ha demostrado que el liderazgo puede amortiguar el impacto de la exposición al combate, al predecir los síntomas de estrés agudo; de modo que el liderazgo positivo reduciría el impacto del combate sobre el estrés agudo en comparación con el de tipo negativo ${ }^{34,35}$. De hecho, existe evidencia sobre el efecto que determinados tipos de liderazgo, como el liderazgo auténtico, se relacionan con un aumento de la moral personal del personal desplegado, de la moral de la unidad, de su cohesión, y en definitiva, de su preparación para el combate ${ }^{36}$. El liderazgo auténtico, a través de sus cuatro dimensiones fundamentales: procesamiento equilibrado, 
moral internalizada, transparencia en las relaciones y autococien$\mathrm{cia}^{37}$, inspira y promueve capacidades psicológicas positivas, que logra que se puedan alcanzar rendimientos que van incluso más allá de las expectativas previstas ${ }^{38}$. Los líderes auténticos generan un entorno de coherencia y ejemplaridad, basado en principios y valores éticos, lo que crea condiciones para aumentar la confianza entre líderes y seguidores. Investigaciones recientes muestran que el liderazgo auténtico se relaciona positivamente con varias variables organizacionales importantes como el capital psicológico colectivo, la satisfacción laboral, la satisfacción de los seguidores con sus líderes, los comportamientos de ciudadanía organizacional, la confianza, el compromiso organizacional y el desempeño laboral ${ }^{39}$, lo que redunda en una mayor moral y preparación del personal, para el cumplimiento de las misiones que le sean encomendadas.

$\mathrm{Y}$ es este concepto, la moral de los combatientes, otro de los aspectos que debemos tener en cuenta en el planeamiento y desarrollo de los despliegues, ya que es un elemento central de las capacidades militare ${ }^{40}$. Su estudio puede favorecer la identificación de fortalezas y debilidades del personal militar, teniendo una relación directa con el desempeño individual o los resultados del grupo en las misiones encomendadas ${ }^{41}$, lo que le convierte en un componente vital en todas las operaciones militares. Entendemos el concepto de moral como el estado positivo de la mente, derivado de un liderazgo político y militar inspirado en un sentido compartido de propósito y valores, bienestar, percepciones de valor y cohesión grupal ${ }^{42}$. Las principales variables que ayudan al mantenimiento y fortalecimiento de la moral son ${ }^{43}$ : confianza en los líderes, cohesión de la unidad, creencia en la tarea, provisión justa de descanso y recreación. Otros autores añaden otros aspectos como la eficiencia en el entrenamiento, el espíritu de cuerpo, buenas condiciones de vida, servicios médicos, promoción profesional y bienestar en general ${ }^{44}$.

De lo que existe evidencia es de que buenos niveles de moral protegen la salud mental del personal de servicio expuesto a peligros o períodos prolongados de estrés, es decir, la moral protege a las tropas contra los desajustes psicológicos ${ }^{40}$. Por lo tanto, los líderes deben ser creativos para mantener un nivel adecuado de actividad y una preparación eficiente para el combate de sus subordinados, respetando el descanso y la calidad de vida de las tropas. Su objetivo principal será siempre el cumplimiento de las misiones, tratando de reducir aquellas vicisitudes que puedan ir erosionando el nivel de moral de los subordinados durante el despliegue, para garantizar que las tropas estarán al máximo de capacidad de respuesta siempre que sea necesario.

Por todo lo expresado anteriormente, el enfoque del presente estudio tendrá un triple objetivo. Por un lado, se analizará el efecto del Liderazgo Auténtico en el Estado Emocional de los soldados, durante un despliegue militar en misión de paz. Por otro, se explorará si existe algún tipo de mediación del nivel de Moral de los subordinados, en la relación del liderazgo con el tipo de emoción. Además, se observará si la acumulación de estrés durante el desarrollo de la misión afecta de algún modo a las relaciones planteadas anteriormente. Para todo ello, se establecerán las siguientes hipótesis de estudio:

Hipótesis 1 (H1): El Liderazgo Auténtico tiene una relación directa sobre las Emociones de los subordinados, de modo que, a mayor presencia de Liderazgo Auténtico, mayor presencia de Emociones Positivas y menor presencia de Emociones Negativas.

Hipótesis 2 (H2): La influencia del Liderazgo Auténtico en el Estado Emocional de los subordinados está mediada por el nivel de Moral de estos.
Hipótesis 3 (H3): Las diferentes fases del despliegue median la relación entre el Liderazgo Auténtico y el Estado Emocional.

\section{MÉTODO}

\section{Muestra}

Se cuenta con un total de 150 militares participantes en la misión de Naciones Unidas en el Líbano (UNIFIL). Dentro de la muestra, es claramente mayoritaria la presencia de los hombres (94\%), frente a la de las mujeres $(6 \%)$. La gran mayoría de los encuestados pertenecen a la escala de Tropa $(69,34 \%)$, mientras que la de Suboficiales $(25,33 \%)$ y la de Oficiales $(5,33 \%)$, tienen menor representación. Respecto a la edad, el 28,8\% del personal corresponde con el personal más joven, comprendido entre 20-30 años; el 51,7\% pertenecen al grupo entre 31-40 años; y el 19,5\% supera este último grupo de edad. En cuanto al estado civil, la mayoría de la muestra está casada o con pareja estable (63.3\%).

\section{Medidas}

Liderazgo Auténtico: se ha utilizado el Authentic Leadership Questionnaire (ALQ), validado en España ${ }^{45}$. El ALQ mide las cuatro dimensiones del modelo teórico: transparencia en las relaciones ("Mi líder dice exactamente lo que quiere decir"), moral internalizada ("Mi líder demuestra creencias que son consistentes con las acciones"), procesamiento equilibrado ("Mi el líder solicita puntos de vista que desafían sus posiciones profundamente arraigadas") y autoconciencia ("Mi líder busca comentarios para mejorar las interacciones con los demás"). La escala de medida consta de un total de 16 ítems, de respuesta tipo Likert de siete puntos, que oscilan entre $0=$ nunca y $7=$ siempre.

Estado Emocional: se ha empleado la adaptación española del Positive and Negative Affect Schedule (PANAS) ${ }^{46}$. El cuestionario consta de 20 ítems, de los cuales 10 pertenecen a la subescala de afecto positivo, y otros 10 a la de afecto negativo. El afecto positivo refleja el punto hasta el cual una persona se siente entusiasmada, activa, alerta, con energía y participación gratificante ("En general, me siento entusiasmado"). El afecto negativo representa una dimensión general de distrés subjetivo y participación desagradable que incluye una variedad de estados emocionales aversivos como disgusto, ira, culpa, miedo y nerviosismo ("Suelo estar irritado"). La intensidad de la emoción se mide a través de una escala tipo Likert de cinco puntos, que oscila entre $1=$ nunca y $5=$ siempre.

Moral: se ha empleado el Cuestionario de Moral para Zonas de Operaciones Militares ${ }^{41}$. Consta de cinco dimensiones: confianza en el mando ("Mi jefe directo nos trata a todos por igual, sin distinciones"), legitimidad de la acción y medios ("Participar en esta misión está siendo una buena oportunidad profesional"), confianza y satisfacción personal ("Me siento capacitado para resolver las dificultades que puedan ocurrir"), cohesión y confianza en la Unidad ("Si tuviera problemas personales, podría contar con la ayuda de mis compañeros") y tiempo libre ("El horario de trabajo a diario es bueno y tengo tiempo para mi"). El cuestionario consta de 36 ítems, de respuesta tipo Likert de cinco puntos, cuya puntuación oscila entre $1=$ totalmente en desacuerdo y $5=$ totalmente de acuerdo. 


\section{Pastor-Álvarez A., Molero-Alonso F., Moriano-León J.A.}

\section{Procedimiento}

El cuestionario fue respondido de manera anónima por los participantes, seleccionados mediante un muestreo aleatorio estratificado, de afijación proporcional. La participación en la prueba tenía un carácter obligatorio, como elemento clave de asesoramiento al mando. Los estratos correspondían a cada una de las Unidades que componían el contingente desplegado. Los participantes fueron informados del uso científico del instrumento, así como de los objetivos del estudio. El cuestionario se aplicó en dos turnos, el primero (T1) cuando el personal se encontraba en la cuarta semana de despliegue, y el segundo (T2) en la vigésima, siendo los mismos participantes en ambos turnos, que se identificaron a través de las 4 últimas cifras de sus números de teléfono móvil. Se utilizaron las instalaciones del comedor de la Base para la cumplimentación de los cuestionarios.

Los datos obtenidos se han analizado utilizando la aplicación estadística IBM SPSS versión 25.0. Para la realización de los estudios de mediación, se ha procedido a utilizar un análisis de mediación simple, mediante la macro de SPSS denominada PROCESS 3.4, utilizando el modelo $\mathrm{n}^{\circ} 4$ de mediación simple ${ }^{47}$, dentro de la estrategia denominada de pasos causales ${ }^{48}$, con el procedimiento de booststrapping que genera intervalos de confianza (al 95\%) que nos permiten tomar decisiones sobre la existencia o no de efectos indirectos. Este modelo analiza la relación entre 1 variable independiente y 1 variable dependiente con la mediación de 1 o más variables mediadoras, desde la perspectiva de una regresión múltiple moderada, cuya base matemática tiene el mismo origen que el de un análisis de la varianza (ANOVA) multivariante.

\section{RESULTADOS}

En primer lugar, se ha procedido a realizar una exploración de los datos de las variables, destinada fundamentalmente a verificar el grado de ajuste a la normalidad estadística de estas variables. Para ello se ha utilizado una visión global de los datos, a partir de estadísticos descriptivos de forma (asimetría y curtosis) y de la prueba de bondad de ajuste de Kolmogorov-Smirnov, en el que dada su alta sensibilidad se consideraría solo el desvío grave $(\mathrm{p}<.01)$. A la par se han calculado todos los estadísticos descriptivos habituales de centralidad (media y mediana) y de variabilidad (desviación estándar, rango observado y rango intercuartil). Los resultados se resumen en la tabla 1.

Tabla 1: Análisis exploratorio y descriptivo. Variables recogidas. $(N=150)$

\begin{tabular}{|c|c|c|c|c|c|c|c|c|}
\hline \multirow{2}{*}{ VARIABLE } & \multicolumn{3}{|c|}{ Exploración: Forma } & \multicolumn{2}{|c|}{ Centralidad } & \multirow[t]{2}{*}{$\begin{array}{l}\text { Rango (Mín. I } \\
\text { Máx.) }\end{array}$} & \multicolumn{2}{|c|}{ Variabilidad } \\
\hline & Asimetría & Curtosis & $\begin{array}{c}\text { Test KS: p } \\
\text { valor }\end{array}$ & Media & Mediana & & $\begin{array}{c}\text { Desviación } \\
\text { estándar }\end{array}$ & Rango intercuartil \\
\hline ALQ - T1 & -0.66 & -0.49 & .067 NS & 65.29 & 70.00 & $12.0 / 96.0$ & 21.33 & 29.75 \\
\hline MORAL - T1 & -0.28 & 0.14 & $.834^{\mathrm{NS}}$ & 15.91 & 16.08 & $7.0 / 21.7$ & 2.74 & 3.58 \\
\hline PANAS POS - T1 & -0.41 & 0.18 & $.355 \mathrm{NS}$ & 40.89 & 41.00 & $22.0 / 50.0$ & 5.43 & 7.00 \\
\hline PANAS NEG - T1 & 0.65 & -0.08 & $.041 *$ & 16.84 & 16.00 & $10.0 / 29.0$ & 4.50 & 7.00 \\
\hline ALQ - T2 & -0.58 & -0.11 & $.405^{\mathrm{NS}}$ & 59.46 & 59.50 & $0.0 / 96.0$ & 23.43 & 31.25 \\
\hline MORAL - T2 & -0.32 & -0.03 & $.458 \mathrm{NS}$ & 15.39 & 15.67 & $7.8 / 21.7$ & 2.86 & 3.21 \\
\hline PANAS POS - T2 & -0.34 & -0.27 & $.493 \mathrm{NS}$ & 40.39 & 40.00 & $23.0 / 50.0$ & 5.79 & 8.00 \\
\hline PANAS NEG - T2 & 0.52 & -0.35 & .099 NS & 17.96 & 18.00 & $10.0 / 30.0$ & 5.00 & 7.25 \\
\hline
\end{tabular}

NS = Desvío no significativo $(\mathrm{p}>.05)$ la variable se distribuye normalmente

$*$ = Desvío significativo pero leve $(\mathrm{p}<.05)$ la variable tiende hacia el modelo normal

La exploración a determinado que los valores de los coeficientes de asimetría y curtosis se encuentran en todas las variables dentro del rango admitido para la tendencia hacia la normalidad $[-1 ;+1]$. Y, finalmente, la prueba de bondad de ajuste de Kolmogorov-Smirnov, nos indica que en todas las variables, excepto una, las diferencia con respecto a la normalidad estadística no alcanzan significación ( $\mathrm{p}>.05$ ); $y$ en la excepción (PANAS NEG en $\mathrm{T} 1)$ el desvío es muy leve $(\mathrm{p}=.041>.01)$. Por tanto, en global podemos concluir que las variables que se han recogido se distribuyen normalmente, o al menos en ninguna de ellas se aprecia un desvío grave con respecto a la normalidad estadística. Como consecuencia de lo anterior, para el estudio de las correlaciones entre las variables podemos aplicar procedimientos estadísticos paramétricos, basados en los coeficientes de correlación de Pearson. 
A continuación, se comprueba si existe relación entre el Liderazgo Auténtico (ALQ), las Emociones Positivas (PANAS POS) o Negativas (PANAS NEG), y la Moral. Se han estimado los coeficientes de correlación de Pearson de ALQ con las otras tres variables tanto en T1 como T2 (tabla 2).

Los resultados muestran que tanto en T1 como en T2 la variable ALQ está significativamente relacionada $(\mathrm{p}<.001)$ con MORAL (intensidad muy alta) y con PANAS POS (intensidad alta), en ambos casos en sentido positivo. Sin embargo, la relación de ALQ con PANAS NEG es de menor intensidad ( $\mathrm{p}<$ .001 ), en sentido negativo. Por su parte, la variable MORAL se relaciona tanto con PANAS POS ( $p<.001$; intensidad alta) en sentido positivo; como con PANAS NEG $(\mathrm{p}<.001$; intensidad moderada), en sentido negativo también.

\section{Análisis de las variables en T1}

Para comprobar si el Liderazgo Auténtico se relaciona directamente con las Emociones Positivas y de forma inversa con las Negativas, como señala nuestra Hipótesis 1, planteamos un modelo de regresión lineal simple para cada una de las opciones. Como se puede observar, el Liderazgo Auténtico tiene una relación significativa directa con las Emociones Positivas ( $p<.001)$, y predice un $20,7 \%$ de su variabilidad (tabla 3 ).

Tabla 2: Matriz de correlaciones bivariadas $(N=150)$

\begin{tabular}{|c|c|c|c|c|c|}
\hline T1 & $\mathbf{A L Q}$ & MORAL & PANAS POS & PANAS NEG & $\mathbf{T} 2$ \\
\hline ALQ & .60 & .59 & .42 & -.24 & ALQ \\
\hline MORAL & .60 & .62 & .50 & -.38 & MORAL \\
\hline PANAS POS & .37 & .51 & .53 & -.47 & PANAS POS \\
\hline PANAS NEG & -.20 & -.33 & -.50 & .50 & PANAS NEG \\
\hline
\end{tabular}

La diagonal señala las correlaciones de las variables en T1 y T2

Los valores por debajo de la diagonal corresponden a T1, y los de por encima a T2

Tabla 3: Regresión lineal simple. Modelo predictivo para Emociones Positivas ( $N=150)$.

\begin{tabular}{|llllllll}
\hline $\mathrm{F}=72.08 ; \mathrm{p}<.001$ & Coef. B & $\begin{array}{c}\text { IC 95\% } \\
\text { de B }\end{array}$ & Estad. & P-Valor & R & $\mathbf{R}^{2}$ & Mejora en $\mathbf{R}^{2}$ \\
\hline Liderazgo Auténtico & 0.33 & $0.27 / 0.39$ & $10.45^{* *}$ & .000 & .439 & .207 & .207 \\
\hline
\end{tabular}

$* * \mathrm{p}<.01$

Respecto a las Emociones Negativas (tabla 4), observamos que el Liderazgo Auténtico tiene una relación significativa negativa con esta variable, de menor intensidad que la anterior, donde predice un $10,6 \%$ de su variabilidad. Por lo tanto, los resultados obtenidos nos permiten confirmar lo establecido en la Hipótesis 1, afirmando que el liderazgo auténtico influye significativamente de forma directa con las emociones positivas de los subordinados, y de forma inversa con las emociones negativas.

Tabla 4: Regresión lineal simple. Modelo predictivo para Emociones Negativas ( $N=150)$.

\begin{tabular}{lccccc}
\hline $\mathrm{F}=36.04 ; \mathrm{p}<.001$ & Coef. B & $\begin{array}{c}\text { IC 95\% } \\
\text { de B }\end{array}$ & Estad. & P-Valor & $\mathbf{R}^{\mathbf{2}}$ \\
\hline Liderazgo Auténtico & -0.19 & $-0.07 /-0.18$ & $-4.40^{* *}$ & .000 & -.203 \\
\hline
\end{tabular}

$* * \mathrm{p}<.01$

A continuación, se procede a realizar el estudio de mediación de la variable Moral, en la relación del Liderazgo Auténtico tanto con las Emociones Positivas como Negativas en T1. En el primer modelo (Fig. 1), se observa que el Liderazgo Auténtico está significativamente relacionado con la Moral $(\mathrm{a}=$ $0.39 ; \mathrm{p}<.001)$. A esto se añade que también existe relación entre Moral y las Emociones Positivas ( $b=0.18$; $p<.001)$, así como un efecto indirecto de esta variable mediadora (IC: 0.02 / 0.10). Dado que además de estas significaciones, el coeficiente b es mayor que c' se concluye que la Moral es una variable mediadora de la relación del Liderazgo Auténtico con las Emociones Positivas. 
Pastor-Álvarez A., Molero-Alonso F., Moriano-León J.A.

Fig. 1: Modelo de Mediación simple. Efecto de la dimensión Liderazgo Auténtico (X) con la mediación de la Moral ( M) sobre las Emociones Positivas $(Y)-(N=150)$

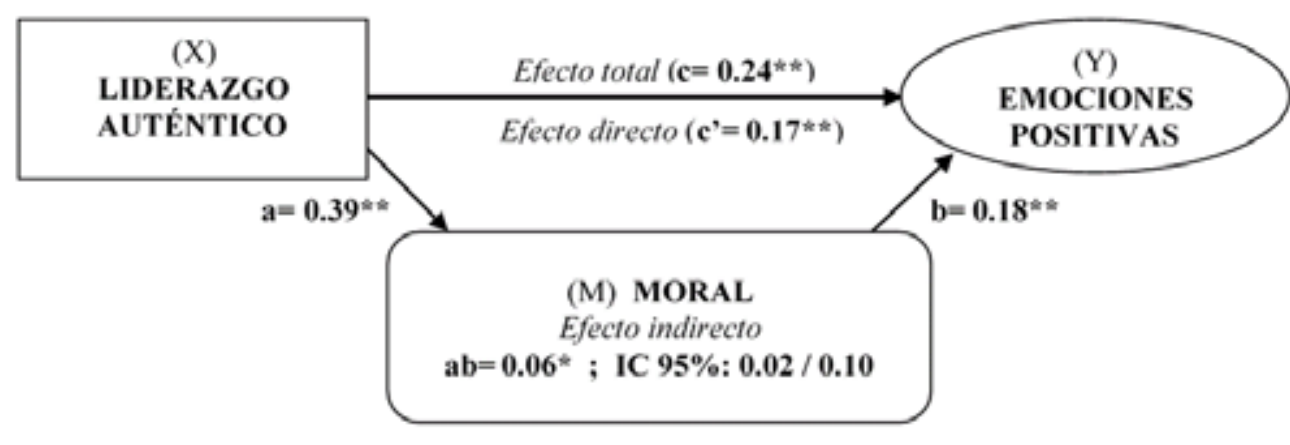

$$
{ }^{\bullet} \mathrm{p}<.05 ;{ }^{\bullet \bullet} \mathrm{p}<.01
$$

En el siguiente modelo de mediación (Fig. 2), se observa que además de la ya conocida relación del Liderazgo Auténtico con la Moral $(a=0.39 ; p<.001)$, existe una relación significativa e inversa de la Moral con las Emociones Negativas ( $b=-0.16$; $p<$ $.001)$, así como un efecto indirecto de esta variable mediadora
(IC: -0.01 / -0.06). Por lo tanto, se concluye que Moral media significativamente la relación analizada, pero su efecto mediador es bajo, puesto que el coeficiente b no tiene un valor superior a c' como habría sido necesario. Según estos resultados la Hipótesis 2 se aceptaría sólo de forma parcial.

Fig. 2: Modelo de Mediación simple. Efecto de la dimensión Liderazgo Auténtico (X) con la mediación de la Moral (M) sobre las Emociones Negativas ( $Y)$ - $(N=150)$

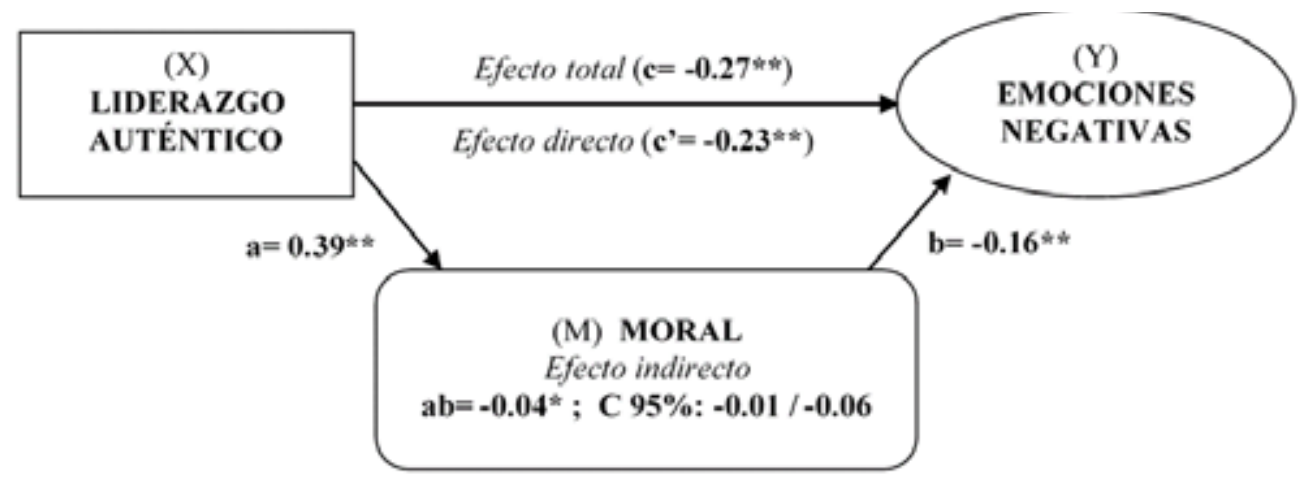

${ }^{*} \mathrm{p}<.05 ;{ }^{* *} \mathrm{p}<.01$

Tabla 5: Análisis comparativo de medidas repetidas. Variación entre Tiempos $T 1$ y $T 2$ ( N=150).

\begin{tabular}{|c|c|c|c|c|c|c|}
\hline \multirow{2}{*}{ Variable } & \multirow{2}{*}{$\begin{array}{c}\text { Medida T1 } \\
\text { Media (D.E.) }\end{array}$} & \multirow{2}{*}{$\begin{array}{c}\text { Medida T2 } \\
\text { Media (D.E.) }\end{array}$} & \multicolumn{2}{|c|}{ T de Student MR } & \multirow{2}{*}{$\begin{array}{c}\text { Tamaño } \\
\text { del efecto: } \\
\mathbf{R}^{2}\end{array}$} & \multirow{2}{*}{$\begin{array}{c}\text { Diferencia Absoluta } \\
\text { Intervalo de confianza al } \\
95 \%\end{array}$} \\
\hline & & & Valor & P-sig & & \\
\hline ALQ & $65.29(21.33)$ & $59.46(23.43)$ & $2.34 *$ & .021 & .035 & $0.90 / 10.76$ \\
\hline MORAL & $15.91(2.74)$ & $15.39(2.86)$ & $1.84 \mathrm{NS}$ & .068 & .022 & ---- \\
\hline PANAS POS & $40.89(5.43)$ & $40.39(5.79)$ & $0.81 \mathrm{NS}$ & .415 & .004 & ---- \\
\hline PANAS NEG & $16.84(4.50)$ & $17.96(5.00)$ & $2.26 *$ & .025 & .033 & $0.14 / 2.10$ \\
\hline
\end{tabular}

$* \mathrm{p}<.05 ; * \mathrm{p}<.01$

\section{Análisis comparativo de las variables en T1 y T2}

Para el estudio de la relación entre el Liderazgo Auténtico y el Estado Emocional de los subordinados (H3), se ha realizado en primer lugar un análisis comparativo de los resultados de las variables de estudio en T1 y T2, a través del contraste $t$-student de medidas repetidas. Los resultados (tabla 5) indican que no hay cambios estadísticamente significativos en la Moral y las Emociones Positivas, pero sí que los hay en la percepción del Liderazgo Auténtico $(p<.05)$ y en las Emociones Negativas ( $\mathrm{p}<.05$ ), aunque son de poca consideración, ya que sus respectivos tamaños del efecto son muy leves $(3.5 \%$ y $3.3 \%$ ).

Los resultados nos indican que si bien no se han obtenido unos tamaños del efecto de consideración, sí que existen diferencias significativas $(\mathrm{p}<.05)$ en las variables de Liderazgo Auténtico y Emociones Negativas, lo que nos lleva a aceptar parcialmente lo establecido en la Hipótesis 3. 


\section{DISCUSIÓN}

El objetivo principal de este estudio ha sido analizar la influencia del Liderazgo Auténtico en el Estado Emocional de los militares desplegados en una misión de paz. Los resultados parecen corroborar la importancia de dicha relación, tanto en aquellos estados emocionales positivos como negativos. Si bien es cierto que la importancia que ha tenido la influencia de este tipo de liderazgo parece tener más relevancia en relación con las emociones positivas expresadas por los subordinados, más que con las negativas. Por lo tanto, podemos seguir afirmando que este modelo de liderazgo ejerce una influencia fundamental como factor de protección $^{27}$, amortiguando los efectos que el estrés derivado del transcurso de la misión pueda ejercer a nivel negativo sobre el bienestar del personal desplegado ${ }^{25}$.

Por otro lado, ha sido también objeto de este trabajo el estudio de la interrelación existente entre el Liderazgo Auténtico y el nivel de Moral de los subordinados durante el despliegue. No solo se ha analizado la solidez de dicha relación, sino que el objetivo fundamental ha sido observar si la variable Moral podía ejercer algún tipo de mediación entre el Liderazgo Auténtico y el Estado Emocional de los subordinados. Los resultados parecen indicar que efectivamente existe una sólida relación de la Moral tanto con el Liderazgo Auténtico como con el Estado Emocional. Sin embargo, en cuanto a la mediación, la Moral sólo ejerce un papel mediador entre el Liderazgo Auténtico y el Estado Emocional cuando las emociones que reportan los subordinados son positivas. En el caso de las emociones negativas, a pesar de la fortaleza de su relación (de carácter inverso) con las otras variables de estudio, la Moral no ha llegado a cumplir los requisitos para poder considerarse como mediadora de la relación entre el liderazgo y las emociones negativas, estableciéndose una mediación parcial ${ }^{49}$. Además, en los estudios posteriores comparando los dos tiempos de evaluación, se ha visto que en el tiempo 2 se había producido una disminución del Liderazgo a la vez que un aumento en las Emociones Negativas, sin que haya habido una modificación significativa en el estado de Moral. Esto nos lleva a hipotetizar que si bien la Moral de los subordinados pueda tener un papel relevante en los niveles más bajos de bienestar o en los sentimientos negativos que se puedan producir a nivel individual o grupal en un despliegue, no tiene que ser condición sine quanum para la aparición de estos en relación con los comportamientos que el líder ejerza sobre sus subordinados. Como ya se ha mencionado, el Liderazgo Auténtico juega un importante papel en el aumento de la Moral tanto a nivel individual como grupal ${ }^{36}$, pero quizás haya que tener en cuenta otros tipos de variables que también puedan ejercer algún tipo de influencia a la hora de experimentar dichas emociones negativas, además de lo ya comentado sobre el liderazgo.

Finalmente, cabe mencionar que no haber encontrado diferencias significativas en las variables de estudio respecto a las diferentes fases de la misión ha sido un resultado inesperado. Sí que se ha evidenciado que el desgaste de la misión puede haber tenido un efecto negativo en cuanto al Estado Emocional de los sujetos, lo que ha redundado en una peor valoración de sus líderes. Dicho hallazgo va en la línea de lo que dice la literatura sobre que los despliegues militares per se pueden afectar a la salud y al bienestar psicológico de los soldados ${ }^{18,19}$, incluso en aquellas operaciones donde el nivel de intensidad y estrés es más moderado ${ }^{20}$. Pero se esperaba que este mismo efecto pudiera haber interferido del mismo modo en una menor presencia de Emociones Positivas y de menor nivel de Moral, en el final de la misión. También debe tenerse en consideración el papel que el avance en los medios de comunicación social ha provocado en el impacto psicológico de los despliegues, ya que algunas de las vicisitudes que mayor deterioro pudieran provocar, como el estar alejado de las familias o las dificultades de comunicación, pudieran estar mitigándose por el desarrollo y puesta a disposición del personal de este tipo de tecnología. En este sentido, podría entenderse que el estrés acumulado de la misión puede haber interferido en cuanto a las Emociones Negativas experimentadas por los subordinados, y las actuaciones y avances en la Moral y bienestar, pueden haber ejercido de factor protector para el personal. Sí es interesante para próximas investigaciones, tener en cuenta que el nivel de Liderazgo Auténtico se ha visto mermado en el transcurso de la misión. Sería interesante poder indagar en las características de las operaciones militares que pueden afectar en este sentido, ya sea por su contexto, duración o condiciones de especial relevancia que vayan en detrimento del Liderazgo o del Estado Emocional del personal desplegado.

El Liderazgo Auténtico ha demostrado ser un modelo eficaz para el futuro en entornos militares ${ }^{50}$, siendo capaz de adaptarse a las vicisitudes de las organizaciones militares, en un entorno cada vez más complejo. Los escenarios futuros de conflicto exigirán que los líderes puedan promover todo el potencial psicológico de sus subordinados, llevándolos a alcanzar unas cotas de rendimiento excepcionales en ambientes inciertos y cambiantes. El camino para lograr dicha excelencia deberá de sustentarse en coherencia, ejemplaridad y valores éticos; para que el compromiso y la confianza tanto con la organización como con los líderes, redunden en mayor eficacia en el cumplimiento de las misiones, y una mejora general en el desempeño labora ${ }^{39}$.

\section{REFERENCIAS}

1. Van der Kolk BA, Herron N, Hostetler A. The History of Trauma in Psychiatry. Psychiatric Clinics of North America, 1994; 17:583-600. http://doi.org/10.1016/ S0193-953X(18)30102-3

2. Sikharulidze G, Van Geloven N, Lelashvili E, Kalandarishvili G, Gugushvili N, Vermetten E. Posttraumatic Stress Disorder and Somatic Complaints in a Deployed Cohort of Georgian Military Personnel: Mediating Effect of Depression and Anxiety. Journal of traumatic stress 2017; 30:626-634. https://doi.org/10.1002/ jts. 22235

3. Laurence JH, Matthews MD. The Oxford handbook of military psychology. Oxford UK: Oxford University Press, 2012.

4. Shea MT, Presseau C, Finley SL, Reddy MK, Spofford C. Different types of combat experiences and associated symptoms in OEF and OIF National Guard and reserve veterans. Psychological Trauma: Theory, Research, Practice, and Policy 2017; 9:19-24. http://doi.org/10.1037/tra0000240

5. Nassif TH, Start AR, Toblin RL, Adler AB. Self-reported mindfulness and soldier health following a combat deployment. Psychological Trauma: Theory, Research, Practice, and Policy 2019; 11(4):466-474. https://doi.org/10.1037/tra0000413

6. Adler AB, Bliese PD, McGurk D, Hoge CW, Castro CA. Battlemind debriefing and battlemind training as early interventions with soldiers returning from Iraq Randomization by platoon. Journal of Consulting and Clinical Psychology, 2009; 77: 928-940. http://dx.doi.org/10.1037/a0016877

7. Wright KM, Cabrera OA, Eckford RD, Adler AB, Bliese PD. The impact of predeployment functional impairment on mental health after combat. Psychological Trauma: Theory, Research, Practice, and Policy 2012; 4:260-269. http://dx.doi. org/10.1037/a0024373 


\section{Pastor-Álvarez A., Molero-Alonso F., Moriano-León J.A.}

8. Adler AB, Britt TW, Castro CA, McGurk D, Bliese PD. Effect of transition home from combat on risk-taking and health- related behaviors. Journal of Traumatic Stress 2011; 24:381-389. http://dx.doi.org/10.1002/jts.20665

9. Wilk JE, Bliese PD, Kim PY, Thomas JL, McGurk D, Hoge CW. Relationship of combat experiences to alcohol misuse among U.S. soldiers returning from the Irac war. Drug and Alcohol Dependence 2010; 108:115-121. http://doi.org/10.1016/j. drugalcdep.2009.12.003

10. Reijnen A, Rademaker AR, Vermetten E, Geuze E. Prevalence of mental health symptoms in Dutch military personnel returning from deployment to Afghanistan A 2-year longitudinal analysis. European Psychiatry 2015; 30:341-346. https://doi. org/10.1016/j.eurpsy.2014.05.003

11. Leroux TC, Hye-Chung K, Dabney A, Wells R. Military deployments and mental health utilization among spouses of active duty service members. Military Medicine 2016; 181(10):1269-1274. https://doi.org/10.7205/MILMED-D-15-00583

12. Martínez JA. Análisis descriptivo del personal militar español asistido por el Servicio de Psicología del ROLE 2 de Herat, Afganistán. Sanidad Militar 2012; 68(3): 163-167. https://dx.doi.org/10.4321/S1887-85712012000300006

13. Borelli JL, Froidevaux NM, Yunusova A, Sbarra DA. Attachment anxiety and trauma history uniquely and interactively predict adjustment during and following deployment among military partners. Journal of Family Psychology 2019; 33(2):226-233. https://doi.org/10.1037/fam0000480

14. Vincenzes KA, Haddock L, Hickman G. The implications of attachment theory for military wives: Effects during a post-deployment period. The Professional Counselor 2014; 4:122-128. http://dx.doi.org/10.15241/kav.4.2.122

15. Krauss SW, Russell DW, Kazman JB, Russell CA, Schuler ER, Deuster PA. Longitudinal effects of deployment, recency of return, and hardiness on mental health symptoms in U.S. Army combat medics. Traumatology 2019; 25(3):216-224. https://doi.org/10.1037/trm0000173

16. Thomas JL, Wilk JE, Riviere LA, McGurk D, Castro CA, Hoge CW. Prevalence of mental health problems and functional impairment among active component and National Guard soldiers 3 and 12 months following combat in Iraq. Archives of General Psychiatry 2010; 67:614-623. http://dx.doi. org/10.1001/archgenpsychiatry.2010.54

17. Bliese PD, Wright KM, Adler AB, Thomas JL, Hoge CW. Timing of postcombat mental health assessments. Psychological Services 2007; 4:141-148. http://dx.doi. org/10.1037/1541-1559.4.3.141

18. Pietrzak E, Pullman S, Cotea C, Nasveld P. Effects of deployment on health behaviours in military forces: A review of longitudinal studies. Journal of Military \& Veterans' Health 2013; 21:14-23.

19. Castro CA, McGurk D. The intensity of combat and behavioral health status. Traumatology 2007; 13: 6-23. https://doi.org/10.1177/1534765607309950

20. Bäccman C, Hjärthag F, Almqvist K. Improved Resiliency and Well Being among Military Personnel in a Swedish Naval Force after a Counter Piracy Operation off the Coast of Somalia. Scandinavian Journal of Psychology 2016; 57:350-358. https://doi.org/10.1111/sjop.12299

21. Seligman ME, Fowler RD. Comprehensive Soldier Fitness and the future of psychology. American Psychologist 2011; 66(1):82-86. https://doi.org/10.1037/ a0021898

22. Cornum R, Matthews MD, Seligman ME. Comprehensive soldier fitness: Building resilience in a challenging institutional context. American Psychologist 2011; 66:4 9. https://doi.org/10.1037/a0021420

23. Adler AB, Litz BT, Bartone PT. The nature of peacekeeping stressors. En : Britt TW, Adler AB (eds.). The psychology of the peacekeeper: Lessons from the field. Westport, CT: Greenwood Publishing Group, 2003:149-168.

24. Brailey K, Vasterling JJ, Proctor SP, Constans JI, Friedman MJ. PTSD symptoms, life events, and unit cohesion in US soldiers: baseline findings from the neurocognition deployment health study. Journal of Traumatic Stress 2007; 20: 495-503. https://doi.org/10.1002/jts.20234

25. Peiró, JM, Rodríguez I. Estrés laboral, liderazgo y salud organizacional. Papeles del psicólogo 2008; 29(1):68-82.

26. Britt TW, Davison J, Bliese PD, Castro CA. How leaders can influence the impact that stressors have on soldiers. Military Medecine 2004; 169(7):541-545. https://doi org/10.7205/MILMED.169.7.541

27. Bardera MP, García M, Pastor A. Gestión de estrés en las Fuerzas Armadas. Revista Del Instituto Español de Estudios Estratégicos 2018; 4:1-24. http://revista. ieee.es/article/view/303

28. Gallus JA, Walsh BM, Van Driel M, Gouge MC, Antolic E. Intolerable Cruelty: A Multilevel Examination of the Impact of Toxic Leadership on U.S. Military Units and Service Members. Military Psychology 2013; 25(6): 588-601. https://doi. org/10.1037/mil0000022
29. Lian H, Ferris LD, Brown DJ. Does taking the good with the bad make things worse? How abusive supervision and leader-member exchange interact to impact need satisfaction and organizational deviance. Organizational Behavior and Human Decision Processes 2012; 117:41-52. https://doi.org/10.1016/j. obhdp.2011.10.003

30. Tepper BJ, Carr JC, Breaux DM, Geider S, Hu C, Hua W. Abusive supervision, intentions to quit, and employees' workplace deviance: A power/dependence analysis. Organizational Behavior and Human Decision Processes 2009; 109:156-167. https://doi.org/10.1016/j.obhdp.2009.03.004

31. Carlson DS, Ferguson M, Perrewé PL, Whitten D. The fallout from abusive supervision: An examination of subordinates and their partners. Personnel Psychology 2011; 64:937-961. https://doi.org/10.1111/j.1744-6570.2011.01232.x

32. Restubog SLD, Scott KL, Zagenczyk TJ. When distress hits home: The role of con- textual factors and psychological distress in predicting employees' responses to abusive supervision. Journal of Applied Psychology 2011; 96:713-729. https://doi.org/10.1037/a0021593

33. Bartone PT. Resilience under military operational stress: Can leaders influence hardiness? Military Psychology 2006; 18:131-148.

34. Mental Health Advisory Team (MHAT-VI). Operation Iraqi Freedom 07-09, Report. Retrieved from U.S. Army Medical Department website: http://www. armymedicine.army.mil/reports

35. Wood MD, Foran HM, Britt TW, Wright KM. The Impact of Benefit Finding and Leadership on Combat-Related PTSD Symptoms. Military Psychology 2012; 24:529-541. https://doi.org/10.1080/08995605.2012.736321

36. McGurk D, Castro CA, Thomas JL, Messer SC, Sinclair RR. Leader Behaviors for Combat Veterans and Noncombat Soldiers. Silver Spring, Md: Walter Reed Army Institute of Research; 2005: 3-4.

37. Walumbwa FO, Avolio BJ, Gardner WL, Wernsing TS, Peterson SJ. Authentic leadership: Development and validation of a theory-based measure. Journal of Management 2008; 34(1): 89-126. https://doi.org/10.1177/0149206307308913

38. Valsania SE, Moriano JA, Molero F. Authentic leadership and intrapreneurial behavior: Cross-level analysis of the mediator effect of organizational identification and empowerment. International Entrepreneurship and Management Journal 2016; 12(1):131-152. https://doi.org/10.1007/s11365-014-0333-4

39. Pastor A, Molero F, Bardera MP, Moriano JA. Authentic leadership and its relationships with work engagement and organizational citizenship behaviors in military units: The role of identification as a mediating variable. Military Psychology 2019; 31(5): 412-424. https://doi.org/10.1080/0899560 5.2019 .1646078

40. Childs S. Soldier Morale: Defending a Core Military Capability. Security Challenges 2016; 12(2): 43-52. https://www.jstor.org/stable/26465606

41. Pastor A, Molero F, Moriano JA. Validation of the Morale Questionnaire for Military Operational Theaters. Revista Acción Psicológica 2019; 16(2):31-42. https://doi.org/10.5944/ap.16.2.24265

42. UK Ministry of Defence. British Defence Doctrine. Joint Doctrine Publication JDP 0-01(3 $3^{\text {rd }}$ Edition) 2008; chapter 4-3

43. Jones E. Morale, Psychological Wellbeing of UK Armed Forces and Entertainment: A Report for The British Forces Foundation. King's Centre for Military Health Research, King's College London 2012; January.

44. Sparrow JH. Second World War 1939-1945 Army, Morale. London: The War Office 1949

45. Moriano JA, Molero F, Lévy-Mangin JP. Liderazgo auténtico. Concepto y validación del cuestionario ALQ en España. Psicothema 2011; 23(2):336-341.

46. Sandín B, Chorot P, Lostao L, Joiner TE, Santed MA, Valiente RM. Escalas PANAS de afecto positivo y negativo: validación factorial y convergencia transcultural. Psicothema, 1999; 11(1): 37-51.

47. Hayes AF. Introduction to Mediation, Moderation and Conditional Process Analysis. A Regression-Based Approach (Methodology in the Social Sciences). New York: The Guilford Press 2013.

48. Baron RM, Kenny DA. The moderator-mediator variables distinction in social psychological research: conceptual, strategic, and statistical considerations. Journal of Personality and Social Psychology 1986; 51(6):1173-1182. https:// doi.org/10.1037/0022-3514.51.6.1173

49. Ato M, Vallejo G. Los efectos de terceras variables en la investigación psicológica. Anales de Psicología 2011; 27(2):550-561.

50. Molero F, Moriano JA. La perspectiva psicosocial del liderazgo en las Fuerzas Armadas. En: Cómo preparar el liderazgo militar futuro. Documento de trabajo del Centro Superior de Estudios de la Defensa 2019; 05:19-39. 